\title{
Impact of hepatitis B on mortality and specific causes of death in adults with and without HIV co-infection in NYC, 2000-2011
}

\author{
J. PINCHOFF*, O. C. TRAN, L. CHEN, K. BORNSCHLEGEL, A. DROBNIK, \\ L. KERSANSKE AND J. FULD \\ New York City Department of Health and Mental Hygiene, Queens, NY, USA
}

Received 2 March 2016; Final revision 1 June 2016; Accepted 19 July 2016;

first published online 11 August 2016

\section{SUMMARY}

High rates of immigration from endemic countries contribute to the high chronic hepatitis $\mathrm{B}$ (HBV) prevalence in New York City (NYC) compared to the United States overall, i.e. about 1 million individuals. We describe the impact of HBV infection on mortality and specific causes of death in NYC. We matched surveillance and vital statistics mortality data collected from 2000 to 2011 by the New York City Department of Health and Mental Hygiene (DOHMH) and analysed demographics and premature deaths (i.e. whether death occurred at $<65$ years) in persons with and without chronic HBV or HIV infection (excluding those with hepatitis C). From 2000 to 2011, a total of 588346 adults died in NYC. Of all decedents, 568753 (97\%) had no report of HIV or HBV, and $4346(0.7 \%)$ had an HBV report. Of HBV-infected decedents, $1074(25 \%)$ were HIV co-infected. Fifty-five percent of HBV mono-infected and 95\% of HBV/ HIV co-infected decedents died prematurely. HBV disproportionately impacts two subgroups: Chinese immigrants and HIV-infected individuals. These two subgroups are geographically clustered in different neighbourhoods of NYC. Tailoring prevention and treatment messages to each group is necessary to reduce the overall burden of HBV in NYC.

Key words: Hepatitis B, HIV/AIDS, mortality, surveillance.

\section{INTRODUCTION}

Hepatitis $\mathrm{B}$ is the most common liver infection in the world $[1,2]$. The hepatitis B virus (HBV) is transmitted through contact with infected blood and other bodily fluids and is 100 times more infectious than human immunodeficiency virus (HIV) [3]. Rate of progression from acute to chronic HBV infection varies by age and immune status at time of infection. Untreated, $20-30 \%$ of chronically infected individuals will develop liver cirrhosis or liver cancer in their lifetime [3]. In 2013, the US HBV-related mortality rate

\footnotetext{
* Author for correspondence: Ms. J. Pinchoff, NYC DOHMH, 42-09 28th Street, Queens, NY 11101, USA. (Email: jpinchoff@gmail.com)
}

was 0.5 deaths $/ 100000$ population, with the highest rate in Asians/Pacific Islanders (APIs) (2.6 deaths/ 100000 population) [4].

The epidemiology of HBV infection varies by geographical area $[1,5]$. Perinatal transmission is the most common cause of HBV infection in high endemic areas ( $\geqslant 8 \%$ prevalence), such as Africa, Southeast Asia, China, and parts of South America, due to inadequate preventive measures during childbirth $[5,6]$. The most common form of HBV transmission in low endemic areas $(<2 \%$ prevalence $)$ is sexual transmission. The United States is considered a low endemic country with an HBV prevalence of $0 \cdot 3-0 \cdot 5 \%$; however, urban centres such as New York City (NYC) experience a substantially higher burden [7]. 
An estimated 1.3 million cases of chronic HBV were diagnosed in the United States from 1974 to 2008. The majority $(95 \%)$ were foreign-born individuals who were infected in their home countries, mainly China, the Philippines, and other HBVendemic Asian countries [8]. High rates of immigration, particularly from China, contribute to the high HBV prevalence in NYC of $\sim 1.2 \%$, over twice the national prevalence [7, 9-12]. An enhanced surveillance study of 180 randomly selected newly diagnosed HBV-infected persons in NYC between 2008 and 2009 identified $67 \%$ as Asian, with $56 \%$ born in China [13]. In addition, $60-80 \%$ of liver cancer cases in APIs are associated with HBV infection [14, 15].

Due to common routes of transmission, persons with HIV/AIDS also have a disproportionate burden of HBV infection [16-18]. NYC experiences higher rates of HIV and HBV infection than the rest of New York State or the United States as a whole, and co-infection with both viruses is associated with premature mortality (death aged $<65$ years) $[5,16]$. Co-infection with both HIV and HBV is associated with more rapid progression of liver damage, greater levels of HBV replication and lower probability of spontaneous loss of $\mathrm{HBV}$ e antigen [19, 20]. A meta-analysis estimated the relative risk of end-stage liver disease to be $\sim 6.14$ and cirrhosis to be 2.07 in those with HBV/HIV co-infection compared to those with HBV mono-infection [21].

Use of antivirals can decelerate HBV disease progression, and screening HBV-infected patients for liver cancer can reduce HBV-related mortality via early cancer diagnosis, treatment, and monitoring $[10,22,23]$. Therefore, understanding the current impact of HBV on mortality at a population level will inform and improve targeted programming to strengthen HBV screening and treatment for at-risk groups. This paper describes the impact of HBV infection, with and without HIV infection (excluding those with hepatitis $\mathrm{C}$ virus), on mortality and examines demographic and socioeconomic risk factors for HBV-related causes of death.

\section{METHODS}

\section{Data matching and analytical population}

The NYC Department of Health and Mental Hygiene (DOHMH) implemented the Centers for Disease Control and Prevention's Program Collaboration and Service Integration initiative to increase data integration and describe interactions across infectious diseases in NYC [24]. As part of this initiative, a crossmatch of disease surveillance registries was conducted, including reports of $\mathrm{HBV}$, hepatitis $\mathrm{C}$ virus (HCV), HIV, tuberculosis, haemoglobin A1c reports, and reportable sexually transmitted diseases received during 2000-2010 and vital statistics mortality data received during 2000-2011 [25-28]. The data matching method has been described previously [26]. Briefly, individuallevel records from separate surveillance registries were linked using deterministic matching methods in SQL and SAS v. 9.2 (SAS Institute Inc., USA). Fourteen matching keys, made up of first name, last name, date of birth and social security number, were used to link the records across datasets. Datasets included all records reported between 2000 and 2011. Information on race/ethnicity, country of birth, and sex was obtained from vital statistics data for this analysis.

The analytical dataset consisted of data as described above for adults aged $\geqslant 18$ years who had an address within the 176 NYC ZIP codes at the time of their death occurring from 2000 to 2011, as identified through vital statistics data. Chronic HBV infection was classified using data from a laboratory or medical provider reported to the NYC DOHMH [29]. Individuals with a positive test for hepatitis $\mathrm{B}$ surface antigen (HBsAg), hepatitis $\mathrm{B}$ e antigen (HBeAg), HBV DNA test, or HBV genotype were considered to have HBV infection [29]. Persons with an acute $\mathrm{HBV}$ infection, indicated by positive hepatitis B core antibody (anti-HBc IgM), were excluded. HIV-infected individuals were defined as persons diagnosed with HIV who were alive as of 1 January 2000 and reported to the DOHMH HIV surveillance registry as of 31 December 2010. HBV/HIV co-infection was identified through the matching with the HIV surveillance registry [25]. Decedents in the dataset were identified based on matches to the vital statistics registry and restricted to persons who died with an NYC place of residence. Those with HCV infection were excluded from analyses, due to the larger prevalence of $\mathrm{HCV}$ and its similar impact on premature mortality and liver cancer in particular.

To explore the association between HBV-related deaths and neighbourhood characteristics, we linked each decedent's ZIP Code Tabulation Area (ZCTA) of residence at the time of death to census data [30]. ZCTAs are created by the U.S. Census Bureau to approximate ZIP codes, which are created by the U.S. Postal Service. ZIP codes that did not convert to a 
ZCTA, but were enclosed within a ZCTA, were modified, where appropriate [30].

Neighbourhood-level poverty, a standardized measure of socioeconomic status, was used to examine disparities in disease burden and HBV-related causes of death [31]. For deaths occurring during 2000-2004, 2005-2009, 2010, and 2011, the poverty data source was the American Community Survey (ACS), 2000, 2007-2011, 2008-2012, and 2009-2013, respectively. Categories were based on the percent of residents with household incomes below $100 \%$ of the Federal Poverty Level (FPL): low poverty $(<10 \%)$, medium $(10$ to $<20 \%$ ), high $(20$ to $<30 \%$ ) and very high poverty $(\geqslant 30 \%)[31]$.

\section{Cause of death}

The DOHMH Bureau of Vital Statistics collects data on all deaths occurring in NYC. Death certificates are completed by clinicians, medical examiners, and funeral directors. The underlying cause for every death reported for NYC residents from 2000 to 2011 was grouped by ICD-10 codes into the following categories for analysis: cardiovascular-related (I00-I01, I05-I06, I09-I13, I20, I21，I24-I28， I31，I33-I35，I38, I40, I42, I44, I46, I48-I51, I60-I64, I67, I69, I70-I74, I77-I78), non-liver cancers (C00-C21, C23-C97), liver cancer (C22), liver-related such as cirrhosis and hepatitis (K70-K77, F10, B16-B19), and HIV/AIDS (B20-B24) [32]. All remaining causes of death were aggregated into 'other' category.

\section{Statistical analysis}

Median age at HBV report, at death, and by cause of death were compared between NYC decedents without HBV or HIV infection and those with HBV mono-infection, and between those with HBV monoinfection and HBV/HIV co-infection, using the Wilcoxon Signed-Rank test. $\chi^{2}$ tests were used to compare the proportion of those with or without HBV report who died prematurely (defined as death at age $<65$ years) or very prematurely (defined as death at age $<50$ years). Demographic factors were examined. To assess the relationship between HBV status and specific causes of death, we constructed multivariable logistic regression models using a binary outcome variable for each category of death. Decedents with HIV mono-infection were excluded from the logistic regression models because premature death from HIV/AIDS tends to occur at a younger age than
HBV-related causes of death, and HBV-related mortality was the primary outcome of interest. All models controlled for sex, age at death (categorized as $<50$, $50-64$ and $\geqslant 65$ years), year of death, race/ethnicity, foreign-born status, and neighbourhood poverty level. Statistical analyses were conducted using SAS. For all analyses, we considered $P<0.05$ to indicate statistical significance.

Four maps were created to assess geographical distribution of poverty, proportion of ZCTA populations born in China, and average age-adjusted HBV-related mortality by ZCTA. NYC DOHMH population estimates by age group and poverty level were obtained from U.S. Census Bureau interpolated intercensal population estimates from 2000 to 2011 . We calculated annual mortality rates per ZCTA using the average number of deaths per year as the numerator and the average population size for each year between 2000 and 2011 in each ZCTA. Mortality rates were adjusted using direct standardization for age at death, grouped into 10 age groups $(<1,1-14,15-24$, 25-34, 35-44, 45-54, 55-64, 65-74, 75-84, $\geqslant 85$ ), and weighted by the US 2000 standard population. The neighbourhood poverty map and percent Chinese-born maps were based on 2010 estimates from the 2010 ACS [33]. Maps were generated using ArcGIS, v. 10.2 [Environmental Systems Research Institute (ESRI) 2011; ArcGIS Desktop, Release 10, USA].

\section{RESULTS}

During 2000-2011, 588346 persons aged $\geqslant 18$ years died in NYC. A total of $23980(4 \cdot 1 \%)$ persons had $\mathrm{HCV}$, and were excluded. Based on match results, after excluding $\mathrm{HCV}$ infections (including 56 with HCV/HBV co-infection), a total of 568753 (97\%) had neither HIV nor HBV infection, $15247(2 \cdot 6 \%)$ were HIV mono-infected, and $3272(0 \cdot 6 \%)$ were HBV mono-infected. Of those with HBV, 1074 (25\%) were co-infected with HIV.

\section{Demographics}

The majority of HBV mono-infected (68\%), HIV mono-infected $(65 \%)$ and HBV/HIV co-infected $(77 \%)$ decedents were male compared to $46 \%$ of decedents with neither infection (Table 1). Of HBV monoinfected decedents, $20 \%$ were non-Hispanic white compared to $54 \%$ of uninfected decedents. In contrast, $37 \%$ of HBV mono-infected decedents were API 
Table 1. Demographic characteristics of decedents aged $\geqslant 18$ years, New York City, 2000-2011

\begin{tabular}{|c|c|c|c|c|c|c|c|c|c|c|}
\hline & \multicolumn{10}{|c|}{ Infection status } \\
\hline & \multicolumn{2}{|c|}{$\begin{array}{l}\text { Neither infection } \\
(N=568753)\end{array}$} & \multicolumn{2}{|c|}{$\begin{array}{l}\text { HBV } \\
\text { mono-infected } \\
(N=3272)\end{array}$} & \multicolumn{2}{|c|}{$\begin{array}{l}\text { HIV } \\
\text { mono-infected } \\
(N=15247)\end{array}$} & \multicolumn{2}{|c|}{$\begin{array}{l}\mathrm{HBV} / \mathrm{HIV} \\
\text { co-infected } \\
(N=1074)\end{array}$} & \multicolumn{2}{|c|}{$\begin{array}{l}\text { HBV infected } \\
\text { total } \\
(N=4346)\end{array}$} \\
\hline & $n$ & $\%$ & $n$ & $n$ & $n$ & $\%$ & $n$ & $\%$ & $n$ & $\%$ \\
\hline \multicolumn{11}{|l|}{ Characteristic } \\
\hline Male & 262960 & 46 & 2211 & 68 & 9977 & 65 & 828 & 77 & 1307 & 30 \\
\hline $\begin{array}{l}\text { Foreign-born } \\
\text { Race/ethnicity }\end{array}$ & 190296 & 34 & 2090 & 65 & 2667 & 18 & 183 & 18 & 2273 & 54 \\
\hline Non-Hispanic white & 303374 & 54 & 650 & 20 & 2435 & 16 & 134 & 13 & 784 & 18 \\
\hline Non-Hispanic black & 141707 & 25 & 927 & 29 & 8189 & 55 & 708 & 67 & 1635 & 38 \\
\hline Hispanic & 86381 & 15 & 443 & 14 & 4115 & 28 & 196 & 19 & 639 & 15 \\
\hline Non-Hispanic API (all) & 28109 & 5 & 1208 & 37 & 140 & 1 & 14 & 1 & 1222 & 29 \\
\hline API US-born & 809 & 3 & 14 & 1 & 18 & 13 & $-*$ & - & - & - \\
\hline API born in China & 14848 & 53 & 882 & 74 & 19 & 14 & $-*$ & - & - & - \\
\hline API born outside China $\dagger$ & 12269 & 42 & 301 & 25 & 101 & 73 & $-*$ & - & - & - \\
\hline \multicolumn{11}{|c|}{ Neighbourhood-based poverty levels ( $\%$ below FPL) } \\
\hline Low $(<10 \%)$ & 121882 & 21 & 392 & 12 & 1232 & 8 & 80 & 7 & 472 & 11 \\
\hline Medium $(10-<20 \%)$ & 205969 & 36 & 1175 & 36 & 3465 & 23 & 257 & 24 & 1432 & 33 \\
\hline High $(20-<30 \%)$ & 138611 & 24 & 1046 & 32 & 4085 & 27 & 303 & 28 & 1349 & 31 \\
\hline Very high $(\geqslant 30 \%)$ & 102152 & 18 & 657 & 20 & 6454 & 42 & 434 & 40 & 1091 & 25 \\
\hline
\end{tabular}

API, Asian/Pacific Islander; FPL, Federal Poverty Level.

* Cells representing $\leqslant 15$ person(s) with an underlying denominator of $\leqslant 500$ persons were suppressed.

$\dagger$ Highest proportions contributed by: Korean (6.2\%), Hong Kong (3·1\%), Philippines $(2 \cdot 5 \%)$ and Bangladesh $(2 \cdot 4 \%)$ 
compared to only $1 \%$ of HBV/HIV co-infected decedents and $5 \%$ of those with neither infection. Of APIs, 99\% were foreign-born, and of the foreign-born APIs, 74\% were born in China. In contrast, the majority $(67 \%)$ of $\mathrm{HBV} / \mathrm{HIV}$ co-infected decedents were non-Hispanic black. Of HIV mono-infected decedents, $55 \%$ were non-Hispanic black, and $1 \%$ were API. Fifty-four percent of those who died in NYC with neither infection were white, $25 \%$ were non-Hispanic black, and 5\% were APIs.

Socioeconomic disparities were observed when comparing neighbourhood poverty levels at time of death by infection status. Eighteen percent of uninfected decedents and $20 \%$ of HBV mono-infected individuals lived in a very high poverty $(\geqslant 30 \%$ below FPL) area at the time of death. In contrast, nearly half of HIV mono-infected (42\%) and HBV/HIV co-infected $(40 \%)$ decedents lived in a very high poverty area.

\section{Premature death}

Compared with New Yorkers with neither infection, the proportions of premature and very premature deaths were significantly higher in HBV monoinfected, HIV mono-infected, and HBV/HIV coinfected decedents (Fig. 1). Of HBV mono-infected decedents, 1803 (55\%) died prematurely, and a subset of $706(21 \%)$ died very prematurely. Of HBV/HIV co-infected decedents, $1060(95 \%)$ died prematurely, and a subset of $767(69 \%)$ died very prematurely. These proportions were significantly higher than those observed in HIV mono-infected decedents ( $92 \%$ and $54 \%$, respectively, $P<0.001$ for each).

The median age at HBV report was significantly younger for HBV/HIV co-infected decedents compared to HBV mono-infected decedents [43 years, interquartile range (IQR) 38-50 vs. 60 years, IQR 49-72) (Table 2). The median age at death for HBV/ HIV co-infected was 46 (IQR 40-52), similar to HIV mono-infected (48, IQR 41-56), and significantly younger compared to HBV mono-infected (63, IQR 52-75) decedents and with New Yorkers with neither infection (79, IQR 66-87) (Table 2).

\section{Causes of death}

In NYC decedents with neither HBV nor HIV infection, the leading causes of death were cardiovascular causes $(48 \%)$ and non-liver cancers $(23 \%)$, with only $1 \%$ dying of liver cancer (Table 3). Of decedents with HBV mono-infection, $27 \%$ died from cardiovascular-related causes, $25 \%$ from non-liver cancer, and $15 \%$ from liver cancer. Of decedents with HIV mono-infection, $62 \%$ died from HIV/ AIDS and 1\% died of liver cancer. For decedents with HBV/HIV co-infection, $66 \%$ of deaths were due to HIV/AIDS and 3\% were due to liver cancer. The median age at death was significantly younger for those with HBV mono-infection compared to those with neither infection in those who died of cardiovascular causes (70 years vs. 82 years), non-liver cancers (62 years $v s .73$ years), and liver cancer (58 years $v s$. 72 years).

The adjusted odds for each cause of death were compared for HBV mono-infected decedents compared to decedents with neither infection, and HIV mono-infected decedents compared to HBV/HIV co-infected decedents (Table 4). All models controlled for sex, age at death, year of death, race/ethnicity, foreign-born status, and neighbourhood level poverty. Compared to decedents with neither infection, the odds of dying of liver cancer in HBV mono-infected individuals were almost 15 times higher [odds ratio (OR) 14.96, 95\% confidence interval (CI) 13.33$16.71]$ and the odds of dying of liver-related noncancer causes were about six times higher (OR 6.03, 95\% CI 5.34-6.84). Compared to HIV mono-infected decedents, HBV/HIV co-infected decedents had over six times higher odds of dying of liver cancer (OR $6 \cdot 20$, 95\% 3.97-9.68), and over two times higher odds of dying of liver-related non-cancer causes of death (OR 2.12, 95\% CI 1·45-3.09). Compared to uninfected decedents, HBV/HIV co-infected decedents had almost five times the odds of dying of liver cancer (OR 4.57, 95\% CI 3.15-6.64). However, the majority of decedents with HIV mono-infection and HBV/ HIV co-infection died of HIV/AIDS (62\% and $66 \%$, respectively).

\section{Geographical patterns}

Because the majority of foreign-born decedents with HBV mono-infection were from China, we examined whether this sub-population was also concentrated in one of NYC's distinct Chinese neighbourhoods. The average age-adjusted mortality rate in HBV monoinfected persons per ZCTA and the proportion of persons born in China by ZCTA are depicted in Figure 2 $(a, b)$. Forty percent of HBV mono-infected decedents resided in one of ten ZCTAs with the highest proportion of persons born in China ( $>25 \%$ population), 


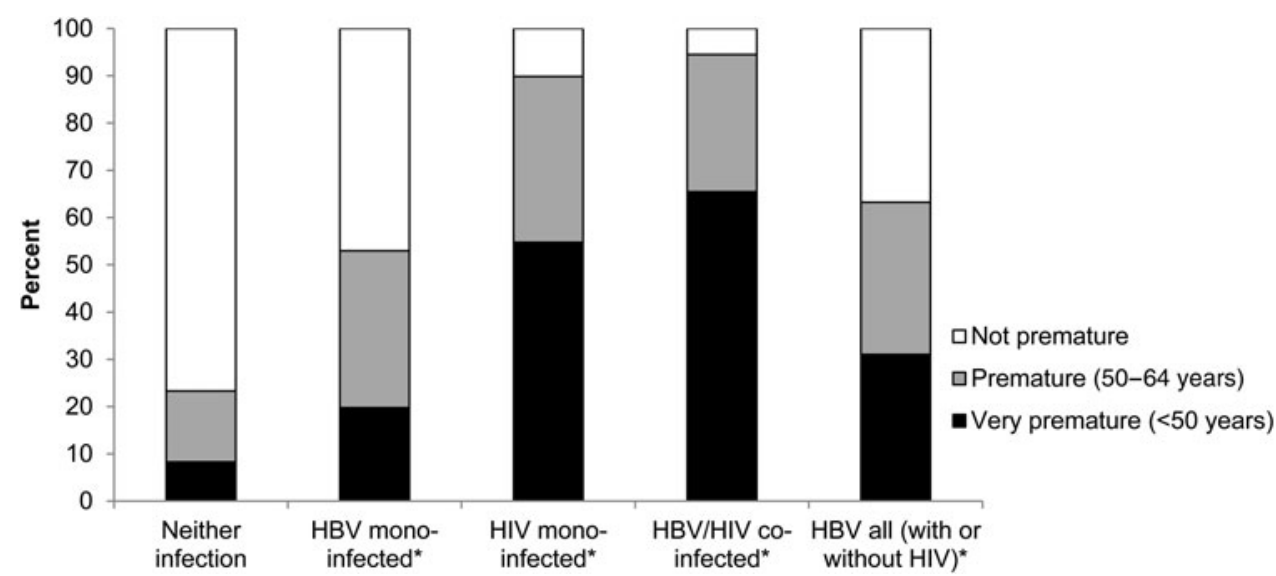

Fig. 1. Proportion of deaths that were not premature, premature (50-64 years) or very premature $(<50$ years) in New York City, stratified by report of HBV, 2000-2011. *Statistical significance $(P<0 \cdot 05)$ when compared to decedents with neither infection.

Table 2. Median age at first report of HBV, age at death by infection status, New York City, 2000-2011

\begin{tabular}{llllll}
\hline \hline & \multicolumn{3}{l}{ Infection status } & & \\
\cline { 2 - 6 } & $\begin{array}{l}\text { Neither } \\
\text { infection }\end{array}$ & $\begin{array}{l}\text { HBV } \\
\text { mono-infected }\end{array}$ & $\begin{array}{l}\text { HIV } \\
\text { mono-infected }\end{array}$ & $\begin{array}{l}\text { HBV/HIV } \\
\text { co-infected }\end{array}$ & $\begin{array}{l}\text { HBV, } \\
\text { all }\end{array}$ \\
\hline $\begin{array}{l}\text { No. of decedents } \\
\begin{array}{c}\text { Age at HBV report } \\
\text { years, median (IQR) }\end{array}\end{array}$ & 568753 & 3272 & 15247 & 1074 & 4346 \\
$\begin{array}{c}\text { Age at death, } \\
\text { years, median (IQR) }\end{array}$ & $79(66-87)$ & $63(52-75)^{*}$ & $48(41-56)^{*}$ & $46(40-52) \dagger$ & $58(47-71)^{*}$ \\
\hline \hline
\end{tabular}

IQR, Interquartile range.

* Statistical significance $(P<0.05)$ compared with decedents with neither infection.

$\dagger$ Statistical significance $(P<0.05)$ compared with decedents with HBV mono-infection.

corresponding with NYC's three Chinatown neighbourhoods.

The average mortality rate in HBV/HIV co-infected persons and poverty levels per ZCTA are depicted in Figure $2(c, d)$. Overall, areas with high mortality rates in HBV/HIV co-infected persons (Fig. 2c) did not overlap with areas exhibiting high mortality rates in HBV mono-infected (Fig. 2a). Rather, areas with high HBV/HIV co-infection mortality rates were concentrated in ZCTAs with high and very high poverty, mainly in northern Manhattan and the Bronx (Fig. 2d).

\section{DISCUSSION}

We identified a disproportionate burden of premature death, particularly due to liver cancer, in individuals with HBV in NYC. Mono-infection with HBV was associated with nearly 15 -fold higher odds of liver cancer-associated mortality compared to neither infection. In contrast, HBV/HIV co-infected individuals were more likely to die at younger ages and from other causes; $66 \%$ of the co-infected decedents died of HIV/AIDS-related causes. However, HBV coinfection appeared to further increase the likelihood of premature death in those with HIV; of these coinfected decedents, risk of dying of liver cancer was increased by over six times. The association between premature death and HBV infection in NYC suggests the need for improved delivery of prevention and care for both persons with and without concurrent HIV infection.

Persons born in countries with intermediate $(2-8 \%)$ or high $(>8 \%)$ prevalence should be screened for HBV and, if negative, vaccinated against $\operatorname{HBV}[22,34]$. Global vaccination rates have increased recently to $82 \%$, and 184 countries now offer HBV vaccination, suggesting the burden of HBV imported into NYC 


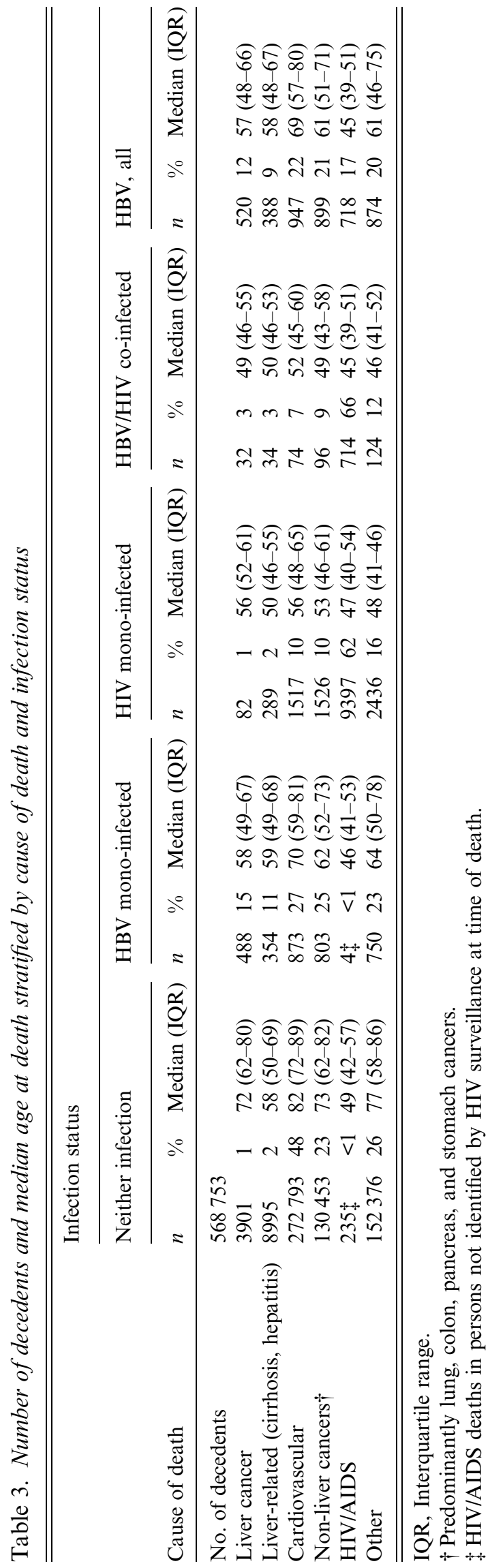

may decline in the future [35]. However, immigrants from endemic countries are often already HBVinfected before entering the United States, and many immigrant APIs remain unvaccinated and unaware that $\mathrm{HBV}$ is vaccine-preventable [5, 7, 34].

Our analysis identified NYC neighbourhoods where screening, prevention and linkage to care campaigns need to be targeted. In NYC, $37 \%$ of HBV monoinfected decedents were APIs, of whom $74 \%$ were born in China, a country with very high HBV prevalence. In addition, $40 \%$ of HBV mono-infected decedents were geographically clustered and resided in one of NYC's three Chinatown neighbourhoods. Prioritizing these high prevalence neighbourhoods with culturally and linguistically tailored educational campaigns and educating clinicians and healthcare providers in high-risk communities on HBV screening recommendations may detect infection earlier and reduce HBV-associated mortality [36].

Despite evidence-based interventions and practice guidelines, HBV mono-infected adults may face barriers to care due to immigration status, lack of health insurance, stigma, language barriers, or lack of awareness [14, 34, 37, 38]. Many HBV-infected individuals are unaware of their infection status and, consequently, do not seek treatment $[14,37,38]$. Lack of education regarding HBV transmission and symptoms are also prevalent in the API population and potential misconceptions and stigma regarding HBV infection hinder HBV test-seeking behaviour [6, 37]. An enduring barrier to care is lack of health insurance for persons with undocumented immigration status; improving health insurance options and access to healthcare for immigrants regardless of their immigration status may improve health outcomes and prevent new infections in this population.

Twenty-five percent of HBV-infected decedents in NYC also had HIV, a common co-infection due to shared risk factors [23]. Co-infection with HIV alters the natural history of HBV and vice versa, leading to more rapid progression of disease. For example, concurrent HBV infection increases the risk of drug-related hepatoxicity and reduces the effectiveness of highly active antiretroviral therapy in HIV-infected individuals [39, 40]. Increased levels of HBV replication associated with HIV infection, accompanied by significantly lower rates of spontaneous reduction in viral replication have also been observed in coinfected individuals [41, 42].

HBV/HIV co-infected individuals were at disproportionate risk for very premature death; of all 
Table 4. Associations between cause-specific deaths and infection status in New York City, 2000-2011

\begin{tabular}{|c|c|c|c|c|c|c|}
\hline \multirow[b]{2}{*}{ Cause of death } & \multicolumn{2}{|c|}{$\begin{array}{l}\text { HBV mono-infected } v s \text {. } \\
\text { neither }\end{array}$} & \multicolumn{2}{|c|}{$\begin{array}{l}\text { HBV/HIV co-infected } \\
v s . \text { neither }\end{array}$} & \multicolumn{2}{|c|}{$\begin{array}{l}\text { HBV/HIV co-infected } \\
\text { vs. HIV mono-infected }\end{array}$} \\
\hline & OR & $95 \% \mathrm{CI}$ & OR & $95 \% \mathrm{CI}$ & OR & $95 \% \mathrm{CI}$ \\
\hline Liver cancer & 14.96 & $13 \cdot 33-16 \cdot 71$ & $4 \cdot 57$ & $3 \cdot 15-6 \cdot 64$ & $6 \cdot 20$ & $3 \cdot 97-9 \cdot 68$ \\
\hline Liver-related non-cancer & $6 \cdot 04$ & $5 \cdot 34-6 \cdot 84$ & $0 \cdot 75$ & $0 \cdot 53-1 \cdot 07$ & $2 \cdot 12$ & $1.45-3.09$ \\
\hline Non-liver cancer & $0 \cdot 90$ & $0 \cdot 83-0 \cdot 98$ & $0 \cdot 31$ & $0 \cdot 25-0 \cdot 39$ & $0 \cdot 89$ & $0 \cdot 71-1 \cdot 12$ \\
\hline Cardiovascular & 0.57 & $0 \cdot 53-0.62$ & $0 \cdot 22$ & $0 \cdot 17-0 \cdot 28$ & $0 \cdot 67$ & $0 \cdot 52-0 \cdot 86$ \\
\hline
\end{tabular}

OR, Odds ratio; CI, confidence interval.

Controlling for sex, age at death, year of death, race/ethnicity, foreign-born status, and ZCTA-level poverty.

HBV/HIV co-infected decedents, 95\% died prematurely and $69 \%$ died very prematurely, proportions that were higher than those with HIV mono-infection. The primary cause of death in co-infected individuals was HIV/AIDS. However, findings suggest that co-infection with HBV may lead to worse HBV outcomes such as liver cancer, as 3\% of co-infected deaths were due to liver cancer compared to $1 \%$ of HIV mono-infected deaths. After controlling for various demographic factors in multivariable logistic regression models, HBV/HIV co-infected adults still had over six times higher odds of dying of liver cancer compared to those with HIV only. This finding is consistent with literature that suggests co-infection with HBV and HIV leads to worse outcomes [18, 19].

HBV/HIV co-infected decedents in NYC were also geographically and demographically distinct from HBV mono-infected decedents. Co-infected decedents were disproportionately non-Hispanic black (67\%) and resided in very high poverty neighbourhoods $(40 \%)$ in northern Manhattan and the Bronx at the time of death. They were also less likely to be foreignborn $(18 \%)$ than HBV mono-infected individuals $(65 \%)$. These patterns follow those for HIV monoinfection, which disproportionately impacts high poverty neighbourhoods. These key demographic differences indicate the need for tailored interventions and messaging.

To prevent HBV infection in people with HIV, efforts to provide HBV vaccination and counselling on reducing transmission risk are recommended and should be strengthened [34, 43]. Additionally, early initiation of antiretroviral therapy has been shown to decrease HBV-associated mortality and sequelae in HBV/HIV co-infected persons [44]. Robust implementation of HIV testing, prevention, and linkage to care programmes can also prevent HIV-related and HBVrelated mortality in persons with or at higher risk for
HBV co-infection, such as people who inject drugs and men who have sex with men [19, 44].

Cardiovascular-related and non-liver cancers were the leading causes of death in New Yorkers with neither HBV nor HIV infection and with HBV monoinfection. However, $15 \%$ of HBV mono-infected individuals died of liver cancer, significantly higher than New Yorkers without HBV (1\%). With antiviral therapy and liver cancer screening, studies suggest that these HBV-attributed deaths can be reduced $[5,10$, 19, 38, 44].

\section{Strengths and limitations}

Strengths of this study included the use of novel datamatching methods to understand the burden of co-infections. Chronic HBV, HIV, and vital statistics registries in NYC were matched, allowing for comparison and analysis of mortality outcomes and specific causes of death for HBV-infected individuals. This is a large, comprehensive dataset of all reported HBV infections in NYC, a major urban centre with a disproportionately high burden of HBV. The matching of these data allows for a unique citywide, population-level analysis that has not been conducted previously. Describing the two distinct populations impacted by HBV will inform citywide HBV prevention and management policy.

This study also has some limitations. Denominators were not available for all demographic and risk groups so that subgroup mortality rates could not be calculated and compared. Errors in death certificates may be present in the data, such as misclassification of underlying cause of death, or biases toward specific causes of death (e.g. HIV/AIDS is always categorized as the underlying cause of death in HIV-infected decedents), may also be present in the data [45]. Vital statistics data are limited to deaths recorded within 
(a)
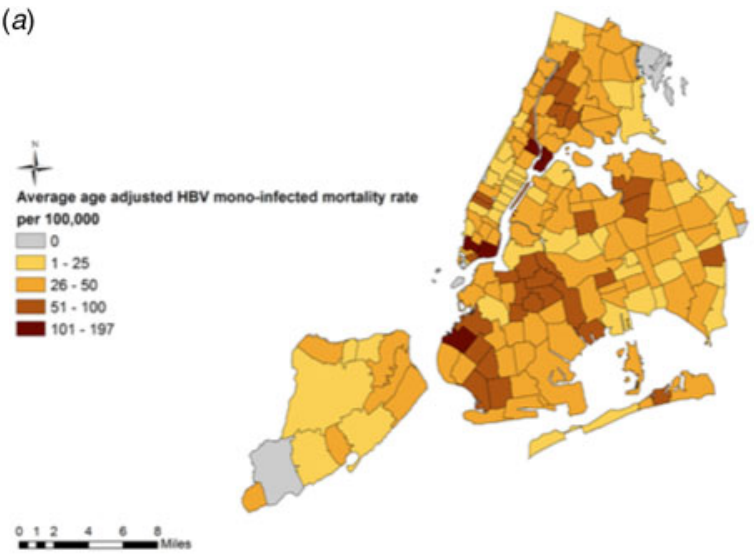

(b)

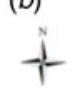

Percent Chinese born

$\square_{0.2}$

$\square_{7.15}^{3-6}$

7.15
$16 \cdot 40$

41.75
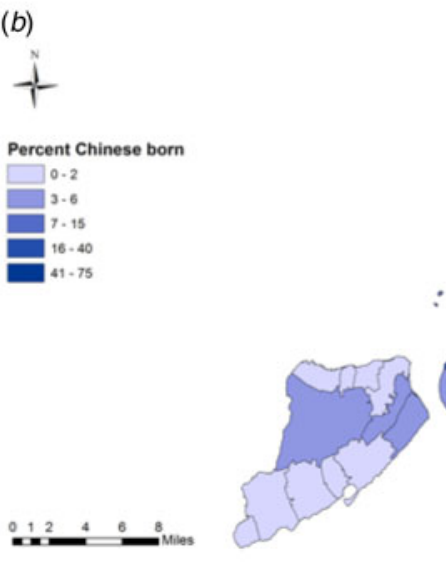

(c)

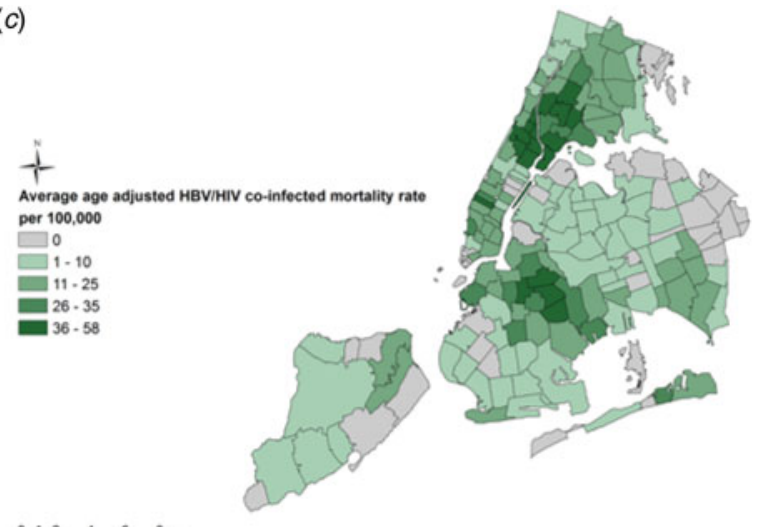

(d)

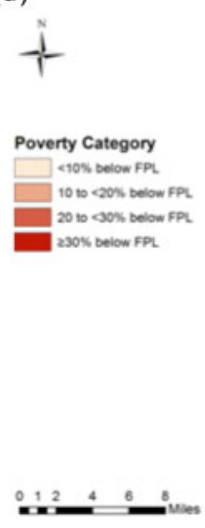

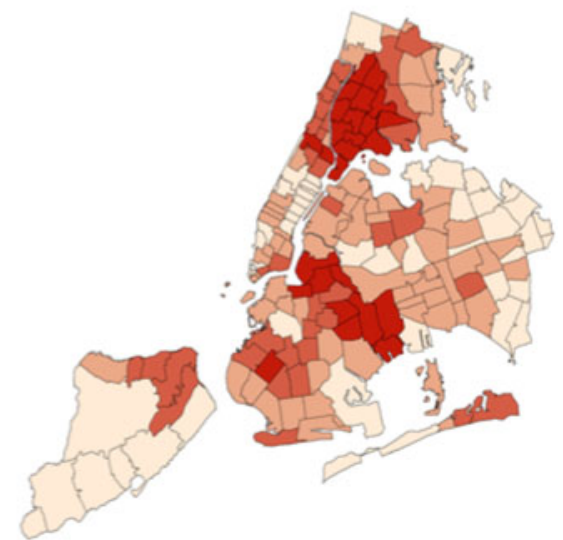

\section{Locator map*}

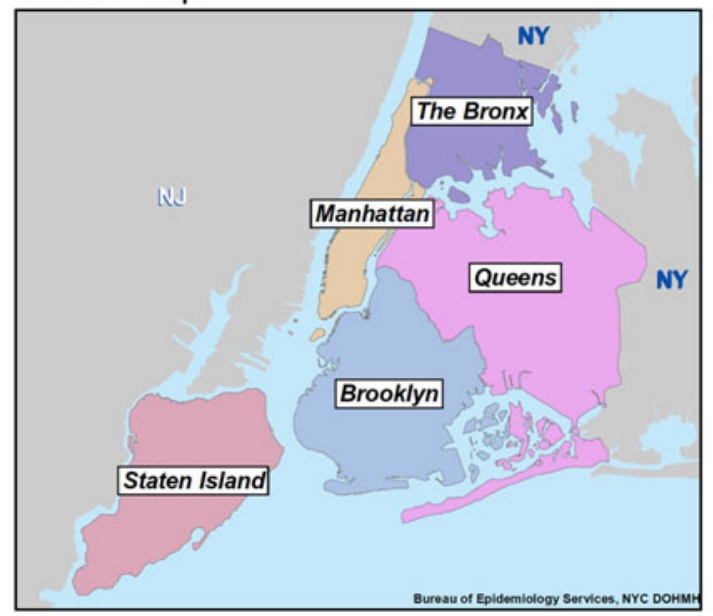

Fig. 2. (a) Average age-standardized mortality rate in HBV mono-infected persons/100 000 from 2000 to 2011, (b) percentage Chinese-born in 2010, (c) average age-standardized mortality rate in HBV/HIV co-infected persons per 100000 from 2000-2011, and (d) poverty category per modified ZCTA in 2010, New York City. * Locator Map [Department of Epidemiology Services, New York City Department of Health. NYC Borough Map (https://www1.nyc.gov/assets/doh/ downloads/pdf/epi/NYC_Borough_Map.pdf)].

NYC, and NYC residents who died outside NYC were not captured in this study [25]. Additionally, some individuals in the chronic HBV dataset may have had acute infection and were no longer infected or may have had a false-positive test result. Persons undiagnosed or unreported with HBV or HIV were 
misclassified as having neither infection, although laboratory reporting on positive diagnostics should be very complete.

\section{CONCLUSIONS}

This study is the first population-level analysis of cause-specific mortality and HBV in NYC. Chinese immigrants and HIV-infected persons need easily accessible services for HBV screening, medical management, early initiation of antiviral or antiretroviral therapy, and liver cancer screening [5, 11, 46]. Through these efforts, incidence of HBV infection (in persons with HIV), liver cancer (mainly in HBV mono-infected persons), premature mortality, and exacerbated HIV disease progression may be decreased [19, 25, 46].

\section{ACKNOWLEDGEMENTS}

The authors thank Sonny Ly and Julie Yuan for conducting the data match, Sharon Greene for her epidemiological guidance, and Wenhui $\mathrm{Li}$ for his assistance with vital statistics data. The authors also thank Sarah Braunstein, James Hadler, Fabienne Laraque, Gretchen Van Wye, and Jay K. Varma for their comments on the manuscript.

\section{DECLARATION OF INTEREST}

None.

\section{REFERENCES}

1. Maclachlan J, Cowie B. Hepatitis B virus epidemiology. Cold Spring Harbor Perspectives in Medicine 2015; 5: a021410.

2. NYC DOHMH. Hepatitis B and C surveillance report: New York City 2013. New York, NY: New York City Department of Health and Mental Hygiene, Division of Disease Control, Bureau of Communicable Diseases, 2015.

3. WHO. Guidelines for the prevention, care and treatment of persons with chronic hepatitis B infection. Geneva, Switzerland, 2015.

4. CDC. Surveillance of viral hepatitis - United States, 2013. United States: Centers for Disease Control and Prevention, Hepatitis DoV, 20 July 2013, 2015 (http:// www.cdc.gov/hepatitis/statistics/2013surveillance/pdfs/ 2013hepsurveillancerpt.pdf).

5. Stasi C, et al. The epidemiological changes of HCV and HBV infections in the era of new antiviral therapies and the anti-HBV vaccine. Journal of Infection and Public Health 2016; 9: 389-395.
6. WHO. Weekly epidemiological record 2009 (http:// www.who.int/wer/2009/wer8440.pdf).

7. Wasley A, et al. The prevalence of Hepatitis B virus infetion in the United States in the era of vaccination. Journal of Infectious Diseases 2010; 202: 192-201.

8. Mitchell T, et al. The increasing burden of imported chronic hepatitis B - United States, 1974-2008. PLoS ONE 2011; 6: e27717.

9. France A, et al. Estimating the prevalence of chronic hepatitis B virus infection-New York City, 2008. Journal of Urban Health 2012; 89: 373-384.

10. Moorman A, et al. Baseline characteristics and mortality among people in care for chronic viral hepatitis: The Chronic Hepatitis Cohort Study. Clinical Infectious Diseases 2009; 56: 40-50.

11. Huang V, et al. Cancer mortality among Asians and Pacific Islanders in New York City, 2001-2010. Journal of Cancer Epidemiology 2013; 2013: 1-9.

12. Hutton D, et al. Cost-effectiveness of screening and vaccinating Asian and Pacific Islander adults for hepatitis B. Annals of Internal Medicine 2007; 147: 460-469.

13. McGibbon E, et al. Surveillance for chronic hepatitis B Virus infection-New York City, June 2008-November 2009. Morbidity and Mortality Weekly Reports 2012; 61: 6-9.

14. Hwang S, et al. Evaluation of hepatitis B and $C$ viral markers: clinical significance in Asian and Caucasian patients with hepatocellular carcinoma in the United States of America. Journal of Gastroenterology and Hepatology 1996; 11: 949-954.

15. Parkin D. The global health burden of infectionassociated cancers in the year 2002. International Journal of Cancer 2006; 118: 3030-3044.

16. Chun H, et al. Hepatitis B virus coinfection negatively impacts HIV outcomes in HIV seroconverters. Journal of Infectious Diseases 2012; 205: 185-193.

17. Rodriguez-Mendez ML, et al. Prevalence, patterns, and course of past hepatitis B virus infection in intravenous drug users with HIV-1 infection. American Journal of Gastroenterology 2000; 95: 1316-1322.

18. Scharschmidt BF, et al. Hepatitis B in patients with HIV infection: relationship to AIDS and patient survival. Annals of Internal Medicine 1992; 117: 837-838.

19. Lacombe K, Rockstroh J. HIV and viral hepatitis coinfections: advances and challenges. Gut 2012; 61: i47-i58.

20. Konopnicki D, et al. Hepatitis B and HIV: prevalence, AIDS progression, response to highly active antiretroviral therapy and increased mortality in the EuroSIDA cohort. AIDS 2005; 19: 593-601.

21. Graham C, et al. Influence of human immunodeficiency virus infection on the course of hepatitis $\mathrm{C}$ virus infection: a meta-analysis. Clinical Infectious Diseases 2001; 33: $562-569$.

22. CDC. Asian \& Pacific Islanders 2015 (http://www.cdc. gov/hepatitis/populations/api.htm).

23. CDC. HIV/AIDS and viral hepatitis 2015 (http://www. cdc.gov/hepatitis/populations/hiv.htm).

24. CDC. Program Collaboration and Service Integration (PCSI) at NCHHSTP Atlanta, GA: Centers for Disease Control and Prevention (updated 5 February 2015) (http://www.cdc.gov/nchhstp/programintegration/). 
25. Drobnik A, et al. Matching HIV, tuberculosis, viral hepatitis, and sexually transmitted diseases surveillance data, 2000-2010: identification of infectious disease syndemics in New York City. Journal of Public Health Management and Practice 2014; 20: 506-512.

26. Pinchoff $\mathbf{J}$, et al. Deaths among people with hepatitis $\mathrm{C}$ in New York City, 2000-2011. Clinical Infectious Diseases 2014; 58: 1047-1054.

27. Prussing C, et al. HIV and viral hepatitis co-infection in New York City, 2000-2010: prevalence and case characteristics. Epidemiology and Infection 2015; 143: 1408-16.

28. Bushnell G, et al. Characteristics and TB treatment outcomes in TB patients with viral hepatitis, New York City, 2000-2010. Epidemiology and Infection 2015; 143: 1972-81.

29. CDC. Surveillance for chronic hepatitsi B virus infection-New York City, June 2008-November 2009. Morbidity and Mortality Weekly Reports 2012; 61: 6-9.

30. NYC DOHMH. Guidance on the use of 2000-2014 interpolated intercensal population estimates. Department of Health and Mental Hygiene, Bureau of Epidemiology Services, 2015.

31. Toprani A, Hadler JL. Selecting and applying a standard area-based socioeconomic status measure for public health data: analysis for New York City. New York City Department of Health and Mental Hygiene, Epi Research Report, 2013, pp. 1-11 (http://wwwl.nyc.gov/assets/doh/ downloads/pdf/epi/epiresearch-SES-measure.pdf).

32. WHO. International Classification of Diseases, Tenth Revision. Geneva, Switzerland, 2004.

33. US Census Bureau. 'Selected characteristics of the foreign-born population by region of birth: Asia' 2007 to 2011 American Community Survey. (http://fact finder.census.gov/faces/tableservices/jsf/pages/productview. xhtml?pid=ACS_14_5YR_S0505\&prodType=table).

34. Weinbaum C, et al. Recommendations for Identification and Public Health Management of Persons with Chronic Hepatitis B Virus Infection. Morbidity and Mortality Weekly Reports 2008; 57(RR08): 1-20.

35. WHO. Global Immunization Coverage: Fact Sheet. July 2016 (http://www.who.int/mediacentre/factsheets/ fs378/en/).

36. Chao S, et al. The Jade Ribbon Campaign: a model program for community outreach and education to prevent liver cancer in Asian Americans. Journal of Immigrant Minority Health 2007; 11: 281-290.

37. Chao S, Chang E, So S. Eliminating the threat of chronic hepatitis B in the Asian and Pacific Islander community: a call to action. Asian Pacific Journal of Cancer Prevention 2009; 10: 507-512.

38. Juon H, et al. Public Health model for prevention of liver cancer among Asian Americans. Journal of Community Health 2008; 33: 199-205.

39. Sulkowski MS, et al. Hepatotoxicity associated with nevirapine or efavirenz-containing antiretroviral therapy: role of hepatitis $\mathrm{C}$ and $\mathrm{B}$ infections. Hepatology 2002; 35: 182-189.

40. Núñez M, et al. Risk factors for severe hepatic injury after introduction of highly active antiretroviral therapy. Journal of Acquired Immune Deficiency Syndromes 2001; 27: 426-431.

41. Hoffman C, Thio C. Clinical implications of HIV and hepatitis B co-infection in Asia and Africa. Lancet Infectious Diseases 2007; 7: 402-409.

42. Oshitani H, et al. Prevalence of hepatitis B antigens in human immunodeficiency virus type 1 seropositive and seronegative pregnant women in Zambia. Transactions of the Royal Society of Tropical Medicine and Hygiene 1996; 90: 235-236.

43. Bureau of HIVIAIDS Prevention and Control, New York City Department of Health and Mental Hygiene. Comprehensive jurisdictional plan for HIV prevention in New York City: 2012-2016. New York, NY, 2012 (http:// www.uchaps.org/documents/NYCJURISDICTIONAL PLANFINAL9-28-12.pdf).

44. Bräu N, et al. Slower fibrosis progression in HIV/HCVcoinfected patients with successful HIV suppression using antiretroviral therapy. Journal of Hepatology 2006; 44: 47-55.

45. CDC. Instruction Manual Part 2a: Procedures for selection of the underlying cause of death for mortality tabulation (https://www.cdc.gov/nchs/data/dvs/2a2008Final. pdf).

46. Kumar R, Singla V, Kacharya S. Impact and management of hepatitis $\mathrm{B}$ and hepatitis $\mathrm{C}$ virus co-infection in HIV patients. Tropical Gastroenterology 2008; 29: 136-147. 